\title{
Extracción de Fe, Mn, Zn, Cu y B en cultivo de pepino (Cucumis sativus L.)
}

\section{Uptake of $\mathrm{Fe}, \mathrm{Mn}, \mathrm{Zn}, \mathrm{Cu}$, and $\mathrm{B}$ in a cucumber (Cucumis sativus L.) crop}
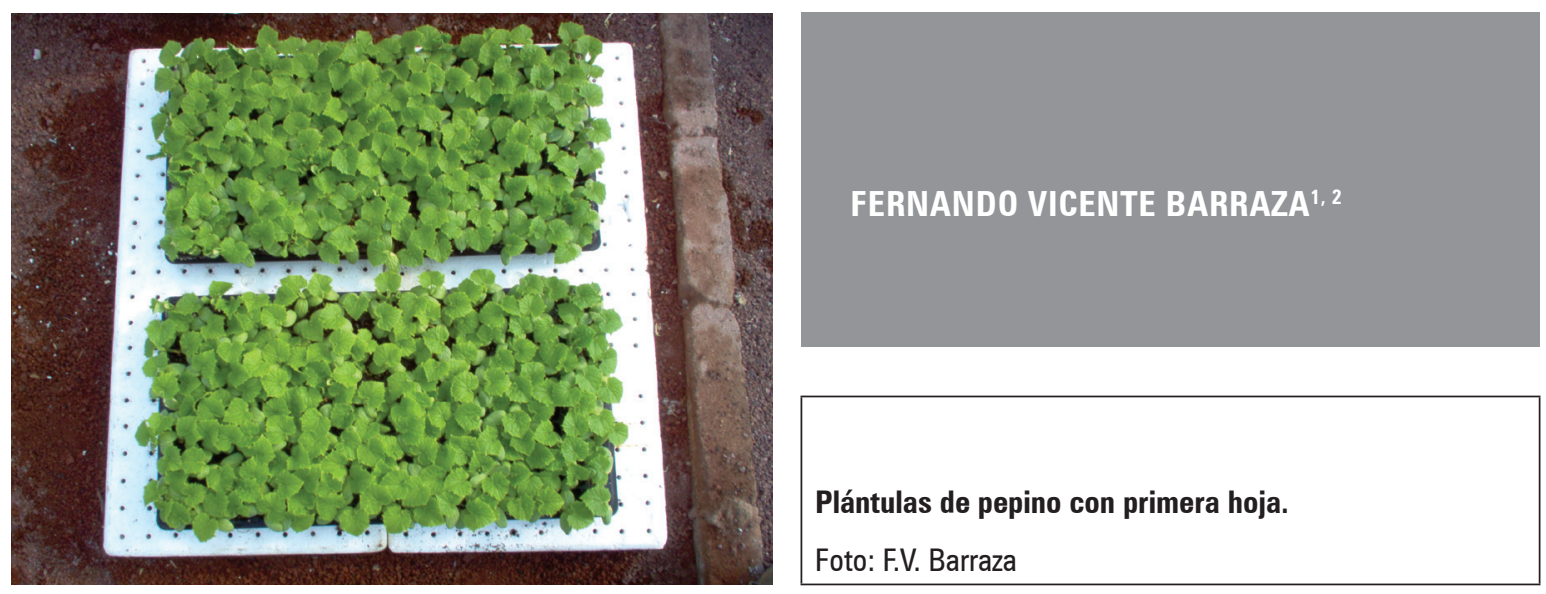

\section{RESUMEN}

El cultivo de pepino, aumenta la acumulación de materia seca y rendimiento cuando se le suministran macro nutrimentos y micro nutrimentos en cantidades adecuadas de acuerdo con su extracción nutrimental. Para el caso de micro nutrimentos, no se cuenta con información exacta sobre las cantidades extraídas por el cultivo cuando se siembra en sistema hidropónico con soluciones nutritivas, lo que puede conducir a aplicaciones excesivas o a deficiencias nutrimentales. Por lo anterior, se hizo una investigación en la Universidad Autónoma Chapingo, México, en condiciones de invernadero y cultivo hidropónico con diseño experimental de bloques al azar y cuatro tratamientos de concentración de la solución nutritiva universal de Steiner 25, 75, 125 y $175 \%$, con el objetivo de determinar el rendimiento, la cantidad de micro nutrimentos Fe, $\mathrm{Cu}, \mathrm{Zn}, \mathrm{Mn}$ y B extraídos por la parte aérea del cultivo y la tasa absoluta de extracción. De acuerdo con los resultados obtenidos, el rendimiento alcanzó $8,20 \mathrm{~kg} /$ planta con la solución nutritiva $175 \%$, mientras las concentraciones 25,75 y $125 \%$ correspondieron a un rendimiento del 40, 78 y $85 \%$, respectivamente, de la solución $175 \%$. El cultivo de 1 t de frutos extrajo 41,74; 34,90; 12,01; 40,84 y 36,91 g de Fe, Cu, Zn, Mn y B, respectivamente.

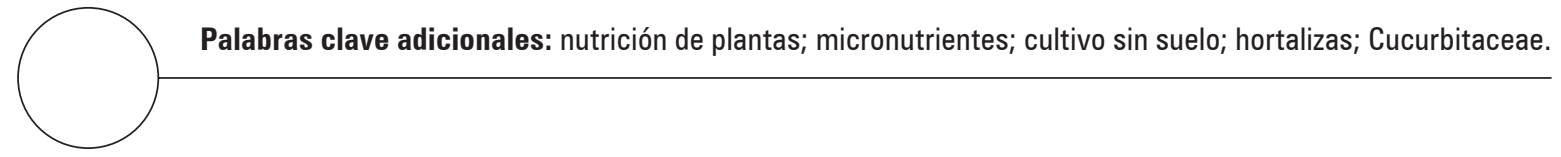

\footnotetext{
ABSTRACT

Cucumber cultivation increases dry matter accumulation and yield when macronutrients and micronutrients are provided at levels that meet their nutritional extraction. In the case of micronutrients, there is no

1 Universidad de Córdoba, Facultad de Ciencias Agrícolas, Montería (Colombia). ORCID Barraza, F.V.: 0000-0002-36712865

2 Autor para correspondencia.fbarraza@correo.unicordoba.edu.co
} 
accurate information on the quantities extracted by this crop when it is sown in a hydroponic system with nutrient solutions, which can lead to excessive applications or nutritional deficiencies. Therefore, this study was conducted at the Universidad Autónoma Chapingo, México, under greenhouse conditions with hydroponic cultivation using a random block experiment design and four treatments consisting of different concentrations of the universal nutrient solution of Steiner: 25, 75, 125 and 175\%, in order to determine the yield, the amounts of Fe, Cu, Zn, Mn and $\mathrm{B}$ extracted by the aerial part of the crop and the absolute extraction rate. According to the results, the yield was $8.20 \mathrm{~kg} /$ plant with the $175 \%$ nutrient solution, and, for the 25,75 and $125 \%$ solutions, the yield was 40 , 78 and $85 \%$, respectively, of the yield obtained with the $175 \%$ solution. For the production of $1 \mathrm{t}$ of fruits, the crop extracted 41.74, 34.90, 12.01, 40.84 and $36.91 \mathrm{~g}$ of Fe, $\mathrm{Cu}, \mathrm{Zn}, \mathrm{Mn}$ and B, respectively.

Additional key words: plant nutrition; trace elements; soilless culture; vegetables; Cucurbitaceae.

Fecha de recepción: 22-05-2018 Aprobado para publicación: 30-11-2018

INTRODUCCIÓN

El pepino, es encuentra dentro de las hortalizas más importantes de la familia de la cucurbitáceas (Eifediyi y Remison, 2010). Su mayor productor es China con 54.315.900 t (Burton, 2017). Requiere suelos fértiles y se ha encontrado significativa respuesta del crecimiento, rendimiento y sus componentes, cuando se aumenta sistemáticamente la cantidad de nutrimentos aplicados (Nwofia et al., 2015).

Con el creciente aumento de la población mundial los terrenos que se destinan a la agricultura son escasos y se presentan efectos adversos para producción de cultivos, ya que principalmente la incorporación de metales pesados procedentes de fertilizantes, pesticidas y actividades industriales ha contribuido a la contaminación y afectan la calidad y seguridad de los alimentos (Savvas et al. 2013). Para el caso de pepino, se presenta la necesidad de mejorar los sistemas de producción, en lo que respecta a la nutrición mineral, lo que ha traído como consecuencia la necesidad de aplicar fertilizantes inorgánicos en grandes cantidades, ya que cuando se realiza el cultivo en suelos infértiles se presenta bajo rendimiento y frutos amargos y deformes, que son rechazados por los consumidores en el mercado (Eifediyi y Remison, 2010; Nwofia et al., 2015).

Debido a lo anterior, se ha intensificado la producción de pepino en sistemas de agricultura protegida, en donde se utilizan invernaderos, genotipos híbridos de alto rendimiento, sistemas hidropónicos y numerosas soluciones nutritivas de diferente composición química para el suministro de nutrimentos, en el caso de México por ejemplo, esta tecnología es importante ya que es el noveno productor mundial de pepinos y pepinillos para el 2017 (FAOSTAT, 2018).

Dentro de las principales ventajas del cultivo de pepino con sistemas hidropónicos es la disminución de los costos de producción y la contaminación de los recursos naturales generados por residuos de fertilizantes. Sin embargo, de acuerdo con Tzerakis et al. (2013), la aplicación de soluciones nutritivas sin conocer sus necesidades nutrimentales pueden ocasionar entradas excesivas o deficitarias de macro nutrimentos y micro nutrimentos, con lo que se afecta considerablemente el rendimiento y calidad.

De acuerdo con Dominy y Bertling (2004) una línea de estudio que se encuentra en desarrollo para el cultivo de pepino es la extracción de micro nutrimentos y el papel que desempeñan en la planta, ya que según indican Tzerakis et al. (2013), se ha encontrado que las deficiencias y excesos de micro nutrimentos, no son perceptibles por los productores hasta que se presentan síntomas externos. Hasta el momento, se le habría restado importancia a la cuantificación de la extracción de micro nutrimentos de este cultivo, debido principalmente, a que los fertilizantes de macro nutrimentos utilizados contienen micro nutrimentos adicionados durante el proceso de manufactura o dichos elementos minerales, se presentan como impurezas y pueden tener una contribución significativa para el suministro del cultivo según como lo indican Moreno et al. (2003). 
Los micro nutrimentos son requeridos por el cultivo en pequeñas cantidades y en concordancia con Küçükyumuk et al. (2014) cumplen funciones importantes en el desempeño fisiológico y metabólico de la planta de pepino y, además, tienen efecto tóxico directo sobre los patógenos y aumentan la rigidez de la pared celular y la integridad de la membrana celular, con lo que se disminuyen las enfermedades en el cultivo.

Para el caso de Fe Vigani et al. (2017) señalan que interviene de manera crucial en la actividad enzimática del metabolismo de la planta; en lo que respecta a $\mathrm{Cu}$, Alaoui-Sossé et al. (2004) y Rouphael et al. (2008) manifiestan que juega un papel importante en la fotosíntesis, respiración, transporte de electrones en cloroplastos y mitocondrias y es cofactor de varias enzimas, como superóxido dismutasa y citocromo c oxidasa; en cuanto a Zn, Küçükyumuk et al. (2014) indican que estabiliza las membranas celulares de las raíces, para aumentar la tolerancia a las infecciones del suelo y desintoxica a la planta de radicales de oxígeno y peróxido de hidrógeno, cuando se da la interacción con los patógenos; respecto a Mn, Maksimović et al. (2016) concluyen que es esencial para el crecimiento y numerosos procesos fisiológicos importantes y de acuerdo con Tabaldi et al. (2007) puede actuar como cofactor de varias enzimas como anhidrasas, deshidrogenasas, oxidasas, peroxidasas y juega un papel importante en la fotosíntesis y la regulación del metabolismo del N, y según Dordas (2008) ayuda a controlar enfermedades en el cultivo, ya que tiene un papel importante en la biosíntesis de lignina; el B, en concordancia con Cikili et al. (2013) y Ramírez et al. (2017), desempeña un papel importante en el metabolismo de ácidos nucleicos, carbohidratos, proteínas, ácido indolacético y fenoles, e interviene en la lignificación de la pared celular, fijación de $\mathrm{N}$, transporte de azúcares, crecimiento de raíces, respiración, germinación del grano de polen y crecimiento del tubo polínico.

Debido a que una fertilización incompleta en cultivo de pepino que no incluya el suministro de micro nutrimentos, ocasiona una drástica reducción en la asimilación fotosintética del $\mathrm{CO}_{2}$ y un severo retardo en el crecimiento del cultivo afectando el rendimiento y calidad (Klamkowski et al., 2011), el objetivo de la presente investigación, fue cuantificar la cantidad de elementos minerales $\mathrm{Fe}, \mathrm{Cu}, \mathrm{Zn}, \mathrm{Mn}$ y B, que extrae la parte aérea del cultivo, para obtener el mayor rendimiento en invernadero con aplicación de la solución nutritiva universal de Steiner, con sistema hidropónico abierto. Dicha solución se distingue por sus relaciones mutuas entre cationes y aniones (Moreno et al., 2015), y en concordancia con Sánchez-del-Castillo et al. (2014) esta condición propicia que las plantas de pepino crezcan sin limitaciones nutricionales, ya que los nutrimentos aplicados están disociados en proporciones y concentraciones que evitan precipitados y antagonismos, por lo cual está siendo ampliamente utilizada por los productores de hortalizas de México.

\section{MATERIALES Y MÉTODOS}

La investigación, se realizó en invernadero entre febrero y junio de 2015 en la Universidad Autónoma Chapingo (México), localizada a 19²9'23' N, 9853'37' W y altitud de $2.250 \mathrm{~m}$. Las condiciones climáticas dentro del invernadero fueron: temperatura promedio diaria de $23^{\circ} \mathrm{C}$, humedad relativa promedio diaria de $67 \%$ y radiación incidente máxima promedio de 12 MJ $\mathrm{m}^{2} \mathrm{~d}^{-1}$. Se utilizó un diseño experimental de bloques al azar con tres réplicas y cuatro tratamientos: $25,75,125$ y $175 \%$ de la solución nutritiva universal de Steiner, que a $100 \%$ de concentración contiene macro nutrimentos (meq L-1) $\mathrm{NO}_{3}^{-} 12 ; \mathrm{H}_{2} \mathrm{PO}_{4}^{-} 1 ; \mathrm{SO}_{4}^{-} 7$; $\mathrm{Ca}^{++} 9 ; \mathrm{Mg}^{++} 4 ; \mathrm{K}^{+} 7$ y micro nutrimentos $\left(\mathrm{mg} \mathrm{L}^{-1}\right)$ $\mathrm{Fe}^{++} 2 ; \mathrm{Cu}^{++} 0,02 ; \mathrm{Zn}^{++} 0,09 ; \mathrm{Mn}^{++} 0,7$ y B- 0,5 (Steiner, 1961).

Se utilizaron plantas del genotipo híbrido Saber ${ }^{\circledR}$ procedentes de semillas sembradas en bandejas de poliestireno expandido con sustrato Peat-Moss ${ }^{\circledR}$ (Comercializadora Hydro Environment, Tlalnepantla, México), trasplantadas a los $25 \mathrm{~d}$ después de la siembra, una plántula a una bolsa de polietileno color negro con $0,06 \mathrm{~m}^{3}$ de sustrato tezontle rojo. En total se utilizaron 156 plantas dispuestas a 1,5 m entre hileras y 0,30 m entre plantas en una superficie de $99 \mathrm{~m}^{2}$. No se efectuaron podas y el tutorado, se hizo en espaldera con malla plástica de cuadrícula de $10 \mathrm{~cm}$.

El suministro de las soluciones nutritivas, se hizo diariamente a cada planta, con sistema de riego por goteo, en sistema hidropónico abierto, sin reutilización de las soluciones nutritivas drenadas, en cantidades y momentos recomendados por Sánchez-del-Castillo y Escalante (1988), de $150 \mathrm{~mL}$, distribuidos en tres riegos de $50 \mathrm{~mL}$, desde el momento del trasplante hasta los $30 \mathrm{~d}$; $300 \mathrm{~mL}$ distribuidos en tres riegos de 100 $\mathrm{mL}$, desde los 31 a $60 \mathrm{~d}$ y $600 \mathrm{~mL}$ distribuidos en tres riegos de $200 \mathrm{~mL}$ desde los 61 hasta $135 \mathrm{~d}$. Entre cada 
riego transcurrió un tiempo de 4 h. Con la aplicación de la solución nutritiva universal de Steiner en sistema hidropónico abierto, según lo indicado por Sánchez-del-Castillo et al. (2014), se obtienen ventajas importantes como el ahorro de agua y fertilizantes y menor impacto ambiental al evitar que grandes cantidades de minerales causen contaminación de suelos y aguas.

Las variables evaluadas, fueron el rendimiento total por planta, extracción de micro nutrimentos por la parte aérea de la planta y tasa absoluta de extracción de micro nutrimentos $\mathrm{Fe}, \mathrm{Cu}, \mathrm{Zn}, \mathrm{Mn}$ y B. El rendimiento total por planta se obtuvo en $\mathrm{kg}$ por planta durante la etapa de fructificación del cultivo, tomando el promedio en tres plantas seleccionadas al azar y marcadas en los tres surcos centrales de cada réplica.

Para determinar la extracción de micro nutrimentos por la parte aérea de la planta, se cuantificó inicialmente el contenido de $\mathrm{Fe}, \mathrm{Cu}, \mathrm{Zn}, \mathrm{Mn}$ y B en la materia seca total, que incluyó hojas, tallos, flores y frutos, para lo cual, se hicieron muestreos cada $15 \mathrm{~d}$ a tres plantas tomadas al azar en las tres hileras centrales de cada repetición, que fueron secadas sin raíces a $65^{\circ} \mathrm{C}$ en estufa Blue-M ${ }^{\circledR}$ POM-326-F (Thermal Product Solutions, New Columbia, PA, USA), hasta obtención de peso constante y posteriormente molidas en molino Wiley Mill Thomas ${ }^{\circledR}$ ED-5. Se tomaron muestras de $100 \mathrm{~g}$ de materia seca, con balanza digital Sartorius 1205 MP $^{\circledR}$ (Sartorius AG, Göttingen, Alemania) y se les realizó análisis de contenido en $\mathrm{mg} \mathrm{kg}^{-1} \mathrm{de} \mathrm{Fe}, \mathrm{Cu}$, Zn, Mn y B en el Laboratorio de Nutrición Vegetal del programa de Edafología e Hidrociencias del Colegio de Posgraduados de Montecillo, México. Fe, Cu, Zn y $\mathrm{Mn}$, se determinaron por digestión ácida y absorción atómica (Mendoza et al., 2015); B se determinó por calcinación y colorimetría con azometina-H (Sadzawka et al., 2004).

A partir de los datos obtenidos de contenido de micro nutrimentos se calculó la extracción de micro nutrimentos por la parte aérea del cultivo $\mathrm{Fe}, \mathrm{Cu}, \mathrm{Zn}, \mathrm{Mn}$ y B de acuerdo con lo indicado por Barraza (2007) mediante la siguiente ecuación:

$\operatorname{Extracción}(\mathrm{mg})=\frac{\left(\text { materia seca }, \mathrm{kg}^{*}\right)\left(\mathrm{mg} \mathrm{kg}^{-1} \text { del nutrimento }\right)}{1000}$ (1)

A las variables rendimiento total por planta y extracción de micro nutrimentos, se les hizo análisis de varianza y pruebas de comparación de medias de Tukey $(P \leq 0,05)$ mediante el software SAS 9.0 (SAS
Institute, 2008). Para conocer en primera instancia el comportamiento de los datos de extracción de micro nutrimentos, se utilizó software Curve Expert versión 1.3 (Hyams, 2003) y de acuerdo con la información obtenida, se aplicó el procedimiento PROC NLIN regresión no lineal con software SAS 9.0 con el modelo logístico: $y=A /\left(1+B \cdot e^{-c x}\right)$. A partir de los modelos obtenidos de extracción de micro nutrimentos se calculó, la tasa absoluta de extracción (TAE), mediante la primera derivada del modelo logístico $\mathrm{y}^{\prime}=\left(\mathrm{A}^{*} \mathrm{~B}^{*}\right.$ $\left.\mathrm{C}^{*} \mathrm{e}^{-\mathrm{Cx}}\right)^{2}$, donde $\mathrm{y}=$ extracción del micro nutrimento; A: máximo valor observado en la extracción del micro nutrimento; B: no tiene significado biológico $\mathrm{y}$ solo toma lugar en el tiempo inicial cuando $\mathrm{x}=0$; $\mathrm{x}=$ días después de la siembra; C: parámetro relacionado con el valor de x para el punto de inflexión; e: constante matemática base del logaritmo natural $=2,718281828$.

\section{RESULTADOS Y DISCUSIÓN}

La extracción de $\mathrm{Fe}, \mathrm{Cu}, \mathrm{Zn}, \mathrm{Mn}$ y B, presentó un comportamiento gráfico sigmoide (Fig. 1), el cual se ajustó al modelo logístico, con coeficientes de determinación cercanos a la unidad (Tab. 1), lo que indica de acuerdo con Song y Qiao (2008) que dichos modelos son razonables y factibles para estimar la extracción de micro nutrimentos a medida que transcurre el ciclo biológico del cultivo y de acuerdo con dichos autores el modelo logístico también tiene importancia en la representación de variables del crecimiento, rendimiento y acumulación de materia seca del cultivo.

Se observó que la extracción de micro nutrimentos en todos los tratamientos desde trasplante hasta aproximadamente los $45 \mathrm{~d}$ fue lenta (Fig. 1), de allí en adelante siguió un periodo de aumento hasta alcanzar su valor máximo y posteriormente ocurrió un periodo de extracción constante a través del tiempo. La tasa absoluta de absorción de los micro nutrimentos (Fig. 2) representó una distribución normal en forma de campana, en concordancia por lo reportado por Vieira et al. (2013), en donde el periodo de mayor extracción de nutrimentos para los tratamientos comprendido entre los 45 y $60 \mathrm{~d}$ posteriores al trasplante. Lo anterior confirma los planteamientos de Sánchez-del-Castillo et al. (2014), en el sentido que la planta modifica el consumo de nutrimentos en función del tiempo que transcurre durante su ciclo biológico, a través de las fases fenológicas que transcurren durante los procesos de crecimiento y 
desarrollo, y que esto se encuentra influenciado por varios factores, entre los que están principalmente las condiciones climáticas, por lo que a partir de los resultados presentados, pueden diseñarse planes de nutrición mineral específicos de acuerdo con cada zona en particular.

Se observa en el tratamiento con solución nutritiva $175 \%$ de concentración, los mayores valores de extracción de micro nutrimentos con respecto a las demás concentraciones utilizadas (Tab. 2) y se encontraron diferencias estadísticas altamente significativas (Tab. 3) lo que señala, según Tzerakis et al. (2013), que la toma de micro nutrimentos por parte del cultivo, estuvo relacionada con la concentración y composición de la solución nutritiva utilizada para cubrir sus necesidades nutrimentales.

Teniendo en cuenta la mayor extracción de micro nutrimentos a medida que se aumentó la concentración de la solución nutritiva del cultivo, confirma lo mencionado por Pso y Nweke (2015), quienes indican que la aplicación de nutrimentos ya sea en forma de fertilizante orgánico o mineral, es la forma más rápida y fácil de aumentar el rendimiento del cultivo de pepino por unidad de superficie, en donde se puede observar que con la concentración al 175\% se obtuvo el mayor valor promedio de rendimiento (Tab. 4).
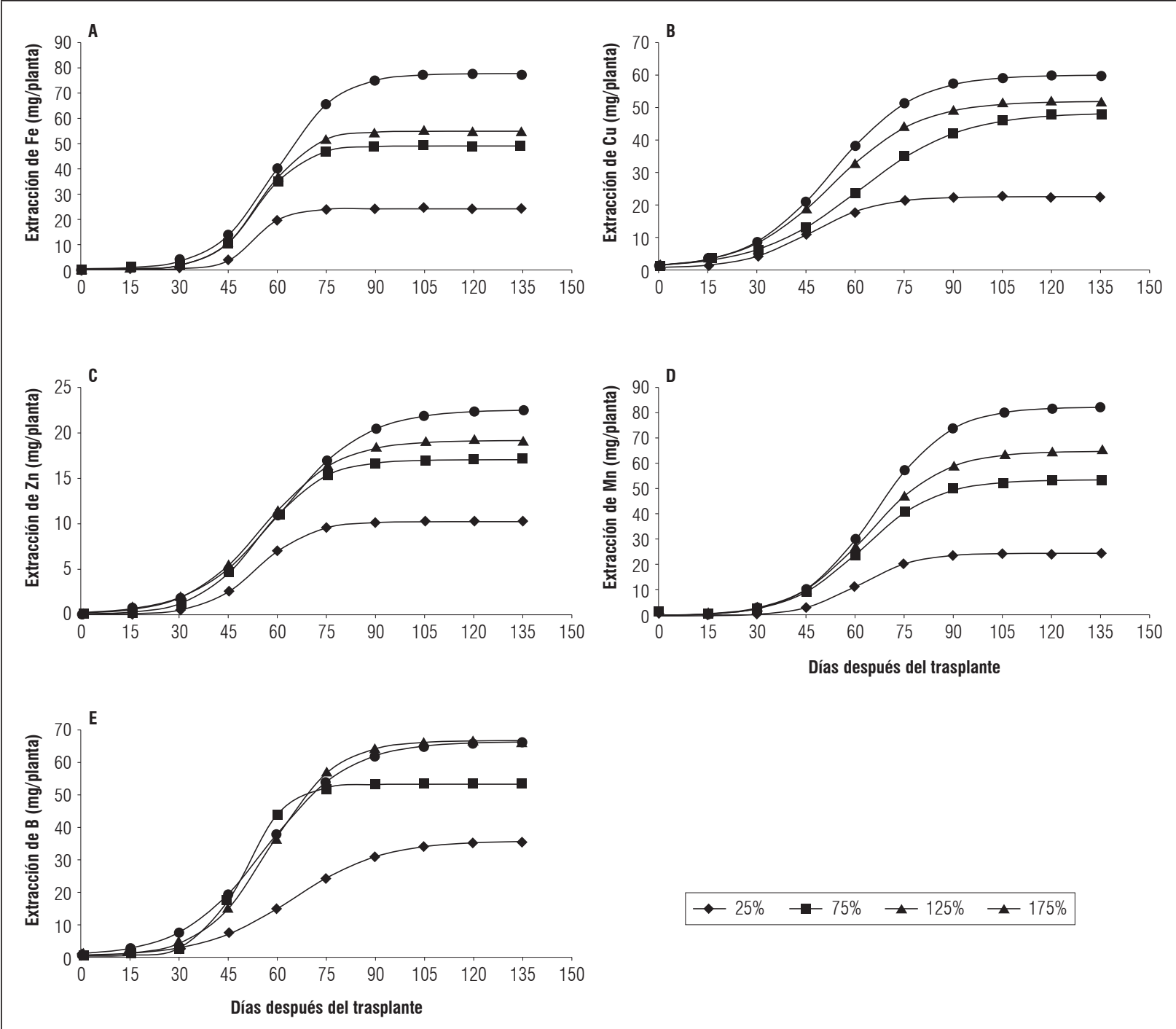

Figura 1. Extracción de micro nutrimentos del cultivo de pepino en cuatro concentraciones de la solución nutritiva universal de Steiner. 
Tabla 1. Modelos matemáticos para estimar extracción de micro nutrimentos en cultivo de pepino.

\begin{tabular}{|c|c|c|}
\hline Concentración de la solución nutritiva (\%) & Modelo matemático & $R^{2^{*}}$ \\
\hline \multicolumn{3}{|c|}{$\mathrm{Fe}$} \\
\hline 25 & $y=23,8519 /\left(1+118438 \mathrm{e}^{-0,2191^{*}} \mathrm{~d}\right)$ & 0,93 \\
\hline 75 & $y=48,7442 /\left(1+2625,6 \mathrm{e}^{-0,1459^{*} \mathrm{~d}}\right)$ & 0,96 \\
\hline 125 & $y=54,5958 /\left(1+2413,8 e^{-0,1400^{*} d}\right)$ & 0,88 \\
\hline 175 & $y=77,4305 /\left(1+615,5 e^{-0,1079^{*}} d\right)$ & 0,99 \\
\hline \multicolumn{3}{|c|}{$\mathrm{Cu}$} \\
\hline 25 & $y=22,2868 /\left(1+97,6 e^{-0,0986^{*}}\right)$ & 0,86 \\
\hline 75 & $y=48,4607 /\left(1+51,4 e^{-0,0645^{*}}\right)$ & 0,94 \\
\hline 125 & $y=51,9787 /\left(1+55,6 e^{-0,0758^{*}}\right)$ & 0,91 \\
\hline 175 & $y=60,1538 /\left(1+68,9 e^{-0,0797^{*}}\right)$ & 0,99 \\
\hline \multicolumn{3}{|c|}{$\mathrm{Zn}$} \\
\hline 25 & $y=10,2553 /\left(1+892,9 e^{-0,1262^{*} d}\right)$ & 0,97 \\
\hline 75 & $y=17,1077 /\left(1+323,1 e^{-0,1062^{*} d}\right)$ & 0,96 \\
\hline 125 & $y=19,2363 /\left(1+133,3 e^{-0,0883^{*} d}\right)$ & 0,95 \\
\hline 175 & $y=22,6475 /\left(1+113,7 e^{-0,0778^{*} d}\right)$ & 0,98 \\
\hline \multicolumn{3}{|c|}{$\mathrm{Mn}$} \\
\hline 25 & $y=24,6712 /\left(1+1168,9 e^{-0,1157^{*} d}\right)$ & 0,93 \\
\hline 75 & $y=53,5976 /\left(1+258,5 e^{-0,0894^{*} d}\right)$ & 0,97 \\
\hline 125 & $y=64,8411 /\left(1+268,4 e^{-0,0878^{*} d}\right)$ & 0,95 \\
\hline 175 & $y=82,2267 /\left(1+402,3 \mathrm{e}^{-0,0908^{*}}\right)$ & 0,99 \\
\hline \multicolumn{3}{|c|}{$\mathrm{B}$} \\
\hline 25 & $y=35,5456 /\left(1+114,9 e^{-0.0730 * d}\right)$ & 0,98 \\
\hline 75 & $y=52,9805 /\left(1+1972,5 e^{-0,1519^{*} d}\right)$ & 0,97 \\
\hline 125 & $y=66,4433 /\left(1+314,3 e^{-0.0994^{*} d}\right)$ & 0,95 \\
\hline 175 & $y=66,1227 /\left(1+84,9 e^{-0,0786^{*}}\right)$ & 0,98 \\
\hline
\end{tabular}

y: extracción (mg/planta); d: días después del trasplante; * : coeficiente de determinación.

Tabla 2. Comparación de medias de la extracción total de micro nutrimentos en la parte aérea de cultivo de pepino.

\begin{tabular}{|c|c|c|c|c|c|}
\hline \multirow{2}{*}{$\begin{array}{c}\text { Concentración } \\
\text { de la solución }\end{array}$} & \multicolumn{5}{|c|}{ Extracción (mg/planta) } \\
\cline { 2 - 6 } & $\mathrm{Fe}$ & $\mathrm{Cu}$ & $\mathrm{Zn}$ & $\mathrm{Mn}$ & $20,61 \mathrm{c}$ \\
\hline $25 \%$ & $17,53 \mathrm{c}$ & $17,47 \mathrm{c}$ & $8,37 \mathrm{c}$ & $40,05 \mathrm{~b}$ & $46,95 \mathrm{~b}$ \\
\hline $75 \%$ & $44,02 \mathrm{~b}$ & $41,83 \mathrm{~b}$ & $15,69 \mathrm{~b}$ & $51,91 \mathrm{~b}$ & $53,130 \mathrm{ab}$ \\
\hline $125 \%$ & $36,64 \mathrm{~b}$ & $38,06 \mathrm{~b}$ & $15,39 \mathrm{~b}$ & $80,50 \mathrm{a}$ & $67,33 \mathrm{a}$ \\
\hline $175 \%$ & $76,03 \mathrm{a}$ & $60,18 \mathrm{a}$ & $21,80 \mathrm{a}$ & 20,90 \\
\hline
\end{tabular}

Promedios con letras distintas en la misma columna, indican diferencia significativa según la prueba de Tukey $(P \leq 0,05)$. 

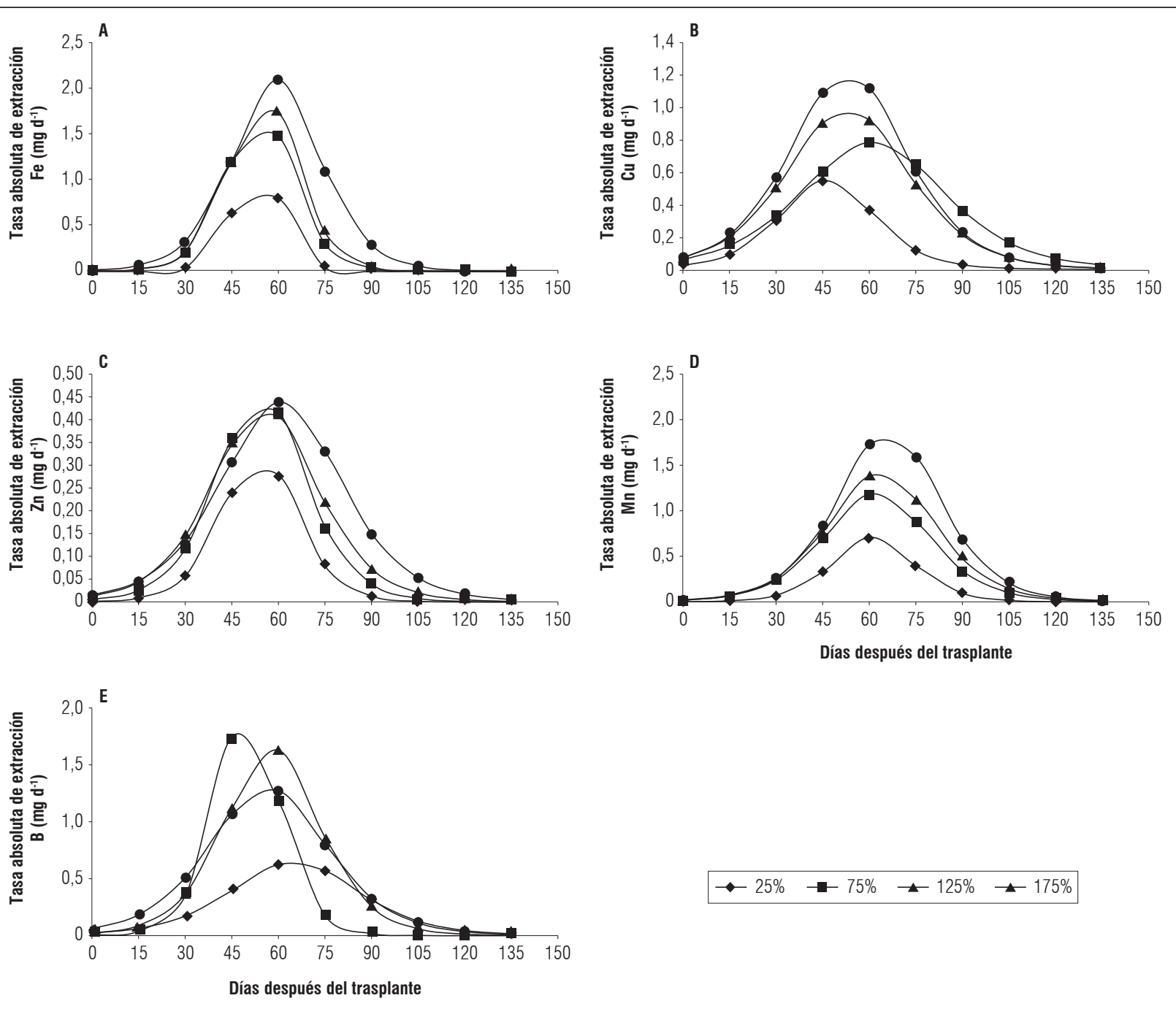

Figura 2. Tasa absoluta de extracción de micro nutrimentos del cultivo de pepino en cuatro concentraciones de la solución nutritiva universal de Steiner.

Tabla 3. Cuadrados medios (CM) del análisis de varianza para extracción de micro nutrimentos (mg/planta) en cultivo de pepino.

\begin{tabular}{|c|c|c|c|c|c|c|}
\hline \multirow{2}{*}{$\mathrm{FV}$} & \multirow{2}{*}{$\mathrm{GL}$} & \multicolumn{5}{|c|}{$\mathrm{CM}$} \\
\hline & & $\mathrm{Fe}$ & $\mathrm{Cu}$ & $\mathrm{Zn}$ & $\mathrm{Mn}$ & B \\
\hline Tratamiento & 3 & $1780,19^{* *}$ & $920,28 * *$ & $90,39 * *$ & $1884,82^{* *}$ & $1132,21^{* *}$ \\
\hline Bloque & 2 & 18,48 & 0,84 & 1,18 & 17,35 & 17,33 \\
\hline Error & 6 & 76,05 & 45,54 & 3,34 & 63,17 & 25,84 \\
\hline CV & & 20,02 & 17,13 & 11,93 & 16,46 & 10,80 \\
\hline
\end{tabular}

FV: fuente de variación; GL: grados de libertad; CV: coeficiente de variación; **: diferencias estadísticas significativas a la $P \leq 0,01$. 


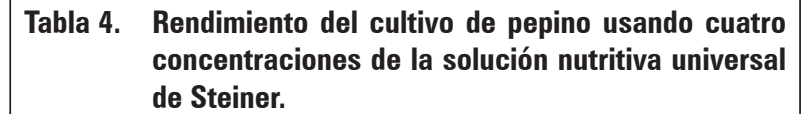

\begin{tabular}{|c|c|}
\hline $\begin{array}{c}\text { Concentración de la solución } \\
\text { nutritiva de Steiner (\%) }\end{array}$ & Rendimiento (kg/planta) \\
\hline 25 & $3,31 \mathrm{c}$ \\
\hline 75 & $6,38 \mathrm{bc}$ \\
\hline 125 & $6,94 \mathrm{~b}$ \\
\hline 175 & $8,20 \mathrm{a}$ \\
\hline
\end{tabular}

Promedios con letras distintas indican a diferencia significativa según la prueba de Tukey $(P \leq 0,05)$.

A la luz de los resultados obtenidos se aprecia la importancia de la extracción nutrimental del cultivo de pepino que de acuerdo con lo indicado por Anjanappa et al. (2012), aprovecha el máximo potencial de los genotipos de alto rendimiento utilizados en la actualidad, así como las bondades del cultivo protegido de esta hortaliza en invernadero y la aplicación de soluciones nutritivas, las cuales proporcionan los nutrimentos requeridos por el cultivo de una manera óptima y propician según Pso y Nweke (2015) mayor crecimiento, sanidad, rendimiento y calidad que se refleja en el mejoramiento sustancial de la eficiencia de fertilizantes y agua y mitigar los impactos al ambiente (Grewal et al., 2011).

Las cantidades de micro nutrimentos requeridas para producir $1 \mathrm{t}$ de frutos (Tab. 5) con la solución nutritiva universal de Steiner al 175\% de concentración, evidencian que el cultivo de pepino presentó mejor respuesta al rendimiento con la mayor aplicación de nutrimentos, lo cual es uno de los aspectos de mayor importancia en la producción de hortalizas en invernadero, con el uso de soluciones nutritivas, lo que de acuerdo con los planteamientos de Moreno et al. (2015) en el cultivo de pepino tiene influencia también en la obtención de frutos de calidad, entendida en términos de apariencia, textura, sabor, aroma, valor nutritivo, constituyentes químicos, propiedades funcionales y ausencia de defectos.

Si bien, el cultivo de pepino se caracteriza de acuerdo con Motior et al. (2011) por una alta extracción de nutrimentos, se ha indicado que los micro nutrimentos son requeridos en pequeñas cantidades en concordancia con lo reportado por Ghehsareh y Samadi (2012), lo cual no resta importancia al papel que juegan dichos elementos en el sistema de oxidación-reducción y en muchos procesos de la actividad celular como
Tabla 5. Cantidades de Fe, Cu, Zn, Mn y B extraídas para producir $1 \mathrm{t}$ de frutos de pepino con la solución nutritiva universal de Steiner a $175 \%$.

\begin{tabular}{|c|c|}
\hline Nutrimento & Extracción $\left(\mathrm{g} \mathrm{t}^{-1}\right)$ \\
\hline $\mathrm{Fe}$ & 41,74 \\
\hline $\mathrm{Cu}$ & 34,90 \\
\hline $\mathrm{Zn}$ & 12,01 \\
\hline $\mathrm{Mn}$ & 40,84 \\
\hline $\mathrm{B}$ & 36,91 \\
\hline
\end{tabular}

coenzimas, de tal manera que su deficiencia puede causar problemas en el metabolismo, crecimiento y rendimiento del cultivo, ya que de acuerdo con Fasaci (2013) y El Sayed et al. (2015) se ha encontrado una alta correlación de la acumulación de materia seca total con la extracción de elementos minerales, tales como Fe, Cu, Zn y Mn.

En concordancia con Ramírez-Pérez et al. (2017), considerando los resultados obtenidos de extracción de micro nutrimentos, en condiciones de invernadero y suministro de solución nutritiva universal de Steiner al $175 \%$, es posible planificar la aplicación de $\mathrm{Fe}, \mathrm{Cu}$, $\mathrm{Zn}, \mathrm{Mn}$ y B de manera más eficiente, de tal modo que se puede lograr la mayor acumulación de materia seca, y a través de los modelos matemáticos de extracción nutrimental (Tab. 1), se pueden cuantificar las cantidades requeridas diariamente de cada micro nutrimento, haciendo posible evitar las aplicaciones excesivas, incrementando la eficiencia de la nutrición mineral en cultivo de pepino.

\section{CONCLUSIONES}

La extracción de micro nutrimentos en el cultivo de pepino aumentó a medida que se aumentó la concentración de la solución nutritiva universal de Steiner.

La aplicación de diferentes de cantidades de micro nutrimentos en el cultivo de pepino, a través de distintas concentraciones de la solución nutritiva universal de Steiner, ocasionó respuesta diferencial en el rendimiento, de tal manera que, con la mayor concentración, se obtuvo el mayor rendimiento, en comparación con los tratamientos de menor concentración.

La mayor extracción de micro nutrimentos, con todas las concentraciones de la solución nutritiva universal 
de Steiner utilizadas, ocurrió entre los 45 y 60 d después de la siembra.

Conflicto de intereses: el manuscrito fue preparado y revisado por el autor, quien declara no tener algún conflicto de interés que coloquen en riesgo la validez de los resultados aquí presentados.

\section{REFERENCIAS BIBLIOGRÁFICAS}

Alaoui-Sossé, B., P. Genet, F. Vinit-Dunand, M.-L. Toussaint, D. Epron y P.-M. Badot. 2004. Effect of copper on growth in cucumber plants (Cucumis sativus L.) and its relationships with carbohydrate accumulation and changes in ion contents. Plant Sci. 166(5), 1213-1218. Doi: 10.1016/j.plantsci.2003.12.032

Anjanappa, M., J. Venkatesha y B. Suresh Kumara. 2012. Dry matter accumulation and uptake of nutrients by cucumber (cv. Hassan Local) as influenced by organic, inorganic and bio-fertilizers. Karnataka J. Agric. Sci. 25(4), 552-554. Doi: 10.1.1.862.3791

Barraza, F.V. 2017. Absorción de N, P, K, Ca y Mg en cultivo de pepino (Cucumis sativus L.) bajo sistema hidropónico. Rev. Colomb. Cienc. Hortic. 11(2), 343-350. Doi: 10.17584/rcch.2017v11i2.7346

Burton, J. 2017. World leaders in cucumber production. En: WorldAtlas,worldatlas.com/articles/the-world-leadersin-cucumber-production.html; consultado: abril de 2018.

Cikili, Y., H. Samet y S. Dursun. 2013. Effects of potassium treatment on alleviation of boron toxicity in cucumber plant (Cucumis sativus L.). Soil-Water J. 2(2), 719-726.

Dominy, A. e I. Bertling. 2004. Manganese, zinc and silicon studies of cucumber (Cucumis sativus) using a miniature hydroponic system. Acta Hortic. 644, 393-398. Doi: 10.17660/ActaHortic.2004.644.52

Dordas, C. 2008. Role of nutrients in controlling plant diseases in sustainable agriculture. A review. Agron. Sustain. Dev. 28(1), 33-46. Doi: 10.1051/agro:2007051

Eifediyi, E.K. y S.U. Remison. 2010. Growth and yield of cucumber (Cucumis sativus L.) as influenced by farmyard manure and inorganic fertilizer. J. Plant Breed. Crop Sci. 2(7), 216-220.

El Sayed, H.E.A., R.O.A. Younis y H.S. Al Othaimen. 2015. Responses of changes in productivity, yield and fruit quality of cucumber (Cucumis sativus L.) plant under bio-and chemical nutrition. Eur. J. Acad. Essays. 2(7), 68-74.

FAOSTAT. 2018. Cultivos. En: http://www.fao.org/faostat/en/\#home; consultado: mayo de 2018.

Fasaei, R.G. 2013. Influence of foliar application of salicylic acid and soil application of humic materials on cucumber and chickpea grown on a nutrient deficient soil. Intl. J. Agri. Crop Sci. 5(21), 2639-2644.

Ghehsareh, A.M. y N. Samadi. 2012. Effect of soil acidification on growth indices and microelements uptake by greenhouse cucumber. Afr. J. Agric. Res. 7(11), 1659-1665.

Grewal, H.S., B. Maheshwari y S.E. Parks. 2011. Water and nutrient use efficiency of a low-cost hydroponic greenhouse for a cucumber crop: an australian case study. Agr. Water Manage. 98(5), 841-846. Doi: 10.1016/j.agwat.2010.12.010

Hyams, D. 2003. Curve Expert 1.3. Hyams Development, Starkville, MS, USA.

Klamkowski, K., W. Treder y A. Tryngiel. 2011. Growth and photosynthetic activity of cucumber as influenced by different fertilization regimes. Ecol. Chem. Eng. 18(1), 35-41.

Küçükyumuk, Z., H. Özgönen, I. Erdal y F. Eraslan. 2014. Effect of zinc and Glomus intraradices on control of Pythium deliense, plant growth parameters and nutrient concentrations of cucumber. Not. Bot. Horti. Agrobo. 42(1), 138-142. Doi: 10.15835/nbha4219346

Maksimović, J.D., M. Mojović y V. Maksimović. 2016. Silicon facilitates manganese phytoextraction by cucumber (Cucumis sativus L.). Zastita Materijala 57(3), 424-429. Doi: 10.5937/ZasMat1603424D

Mendoza, B., L. Almao, L.M. Marcó y V. Rodríguez. 2015. Evaluación de dos métodos de digestión ácida en el análisis de tejido foliar de caña (Saccharum officinarum L.). Ciencia y Tecnología. 7(2), 9-20. Doi: 10.18779/ cyt.v7i2.98

Moreno, D.A., G. Víllora y L. Romero. 2003. Variations in fruit micronutrient contents associated with fertilization of cucumber with macronutrients. Sci. Hort. 97(2), 121-127. Doi: 10.1016/S0304-4238(02)00147-4

Moreno, D., B. Hernández, J. Barrios, A. Ibáñez, W. Cruz y R. Berdeja. 2015. Calidad poscosecha de frutos de pepino cultivados con diferente solución nutritiva. Rev. Mex. Cienc. Agric. 6(3), 637-643. Doi: 10.29312/ remexca.v6i3.648

Motior, M.R., A.S. Abdou, F.H. Al Darwish, K.A. El-Tarabily, M.A. Awad, F. Golam y M. Sofian-Azirun. 2011. Influence of elemental sulfur on nutrient uptake, yield and quality of cucumber grown in sandy calcareous soil. Aust. J. Crop Sci. 5(12), 1610-1615.

Nwofia, G.E., A.N. Amajuoyi y E.U. Mbah. 2015. Response of three cucumber varieties (Cucumis sativus L.) to planting season and NPK fertilizer rates in lowland humid tropics: sex expression, yield and Inter-relationships between yield and associated traits. Int. J. Agric. For. 5(1), 30-37.

Pso, O. y I. Nweke. 2015. Effect of poultry manure and mineral fertilizer on the growth performance and quality 
of cucumber fruits. J. Exp. Biol. Agric. Sci. 3(4), 362367. Doi: 10.18006/2015.3(4).362.367

Ramírez-Pérez, L., A.B. Morales-Díaz, K. de Alba-Romenus, S. González-Morales, A. Benavides-Mendoza y A. Juárez-Maldonado. 2017. Determination of micronutrient accumulation in greenhouse cucumber crop using a modelling approach. Agronomy 7(4), 79-96. Doi: 10.3390/agronomy7040079

Rouphael, Y., M. Cardarelli, E. Rea y G. Colla. 2008. Grafting of cucumber as a means to minimize copper toxicity. Environ. Exp. Bot. 63(1-3), 49-58. Doi: 10.1016/j. envexpbot.2007.10.015

Sadzawka, A., R. Grez Z., M.A. Carrasco y M.L. Mora. 2004. Métodos de análisis de tejidos vegetales. En: Comisión de Normalización y Acreditación-Sociedad Chilena de la Ciencia del Suelo; Instituto de Investigaciones Agropecuarias, Santiago.

SAS Institute Inc. 2008. SAS user's guide: Statistical analysis system. Version 9.0. SAS Institute, Cary, NC.

Song, W. y X. Qiao. 2008. A regression model of dry matter accumulation for solar greenhouse cucumber. pp. 1346-1352. En: Li, D. (eds.). Computer and computing technologies in agriculture. Vol. II. CCTA 2007. The International Federation for Information Processing. Vol 259. Springer, Boston, MA.

Steiner, A. 1961. A universal method for preparing nutrient solutions of a certain desired composition. Plant Soil 15(2), 134-154. Doi: 10.1007/BF01347224

Sánchez-del-Castillo, F., L. González-Molina, E.C. Moreno-Pérez, J. Pineda-Pineda y E.C. Reyes-González. 2014. Dinámica nutrimental y rendimiento de pepino cultivado en hidroponía con y sin recirculación de la solución nutritiva. Rev. Fitotec. Mex. 37(3), 261-269.

Sánchez-del-Castillo, F. y E. Escalante. 1988. Hidroponia. 3a ed. Imprenta Universitaria de la Universidad Autónoma Chapingo, Texcoco, México. pp. 99-151.

Savvas, D., G. Ntatsi y P. Barouchas. 2013. Impact of grafting and rootstock genotype on cation uptake by cucumber (Cucumis sativus L.) exposed to Cd or Ni stress. Sci. Hortic. 149, 86-96. Doi: 10.1016/j.scienta.2012.06.030

Tabaldi, L.A., R. Ruppenthal, D. Cargnelutti, V.M. Morsch, L.B. Pereira y R.M.C. Schetinger. 2007. Effects of metal elements on acid phosphatase activity in cucumber (Cucumis sativus L.) seedlings. Environ. Exp. Bot. 59(1), 43-48. Doi: 10.1016/j.envexpbot.2005.10.009

Tzerakis, C., D. Savvas, N. Sigrimis y G. Mavrogiannopoulos. 2013. Uptake of $\mathrm{Mn}$ and $\mathrm{Zn}$ by cucumber grown in closed hydroponic systems as influenced by the $\mathrm{Mn}$ and $\mathrm{Zn}$ concentrations in the supplied nutrient solution. HortScience 48(3), 373-379. Doi: 10.21273/ HORTSCI.48.3.373

Vieira Neto, J., F.O.G. Menezes Júnior y P.A. Souza Gonçalves. 2013. Produção e curva de crescimento de pepineiros para conserva em manejo convencional e com controle alternativo de pragas. Rev. Ciênc. Agrovet. $12(3), 229-237$.

Vigani, G., D. Di Silvestre, A.M. Agresta, S. Donnini, P. Mauri, C. Gehl, F. Bittner e I. Murgia 2017. Molybdenum and iron mutually impact their homeostasis in cucumber (Cucumis sativus L.) plants. New Phytol. 213(3), 1222-1241. Doi: https://doi. org/10.1111/nph.14214 